\title{
openheart Characterisation of the patients with suspected heart failure: experience from the SHEAF registry
}

\author{
Pankaj Garg (D) , ${ }^{1}$ Ahmed Dakshi, ${ }^{2}$ Hosamadin Assadi (i) , ${ }^{1}$ Andrew J Swift, ${ }^{3}$ \\ Umna Naveed, ${ }^{1}$ Graham Fent, ${ }^{2}$ Nigel Lewis, ${ }^{1}$ Dominic Rogers, ${ }^{2}$ \\ Athanasios Charalampopoulos, ${ }^{2}$ Abdallah Al-Mohammad (1) 1,2
}

\begin{abstract}
- Additional material is published online only. To view please visit the journal online (http://dx.doi.org/10.1136/ openhrt-2020-001448).
\end{abstract}

To cite: Garg P, Dakshi A, Assadi $\mathrm{H}$, et al. Characterisation of the patients with suspected heart failure: experience from the SHEAF registry. Open Heart 2021;8:e01448. doi:10.1136/ openhrt-2020-001448

Received 16 September 2020 Revised 2 December 2020 Accepted 14 December 2020
Check for updates

(c) Author(s) (or their employer(s)) 2021. Re-use permitted under CC BY-NC. No commercial re-use. See rights and permissions. Published by BMJ.

${ }^{1}$ IICD, The University of Sheffield, Sheffield, UK

${ }^{2}$ Cardiology, Sheffield Teaching Hospitals NHS Foundation Trust, Sheffield, UK

${ }^{3}$ Academic Unit of Radiology, The University of Sheffield, Sheffield, UK

Correspondence to Professor Abdallah AlMohammad; abdallah.almohammad@nhs.net

\section{ABSTRACT}

Objectives To characterise and risk-stratify patients presenting to a heart failure (HF) clinic according to the National Institute for health and Care Excellence (NICE) algorithm.

Methods This is an observational study of prospectively collected data in the Sheffield HEArt Failure registry of consecutive patients with suspected HF between April 2012 and January 2020. Outcome was defined as allcause mortality.

Results 6144 patients were enrolled: $71 \%$ had HF and $29 \%$ had no HF. Patients with N-terminal pro-brain-type natriuretic peptide (NT-proBNP) $>2000 \mathrm{pg} / \mathrm{mL}$ were more likely to have HF than those with NT-proBNP of $400-2000 \mathrm{pg} / \mathrm{mL}$ (92\% vs 64\%, respectively). Frequency of HF phenotypes include: HF with preserved ejection fraction (HFpEF) (33\%), HF with reduced ejection fraction (HFrEF) (29\%), HF due to valvular heart disease (4\%), HF due to pulmonary hypertension (5\%) and HF due to right ventricular systolic dysfunction (1\%). There were 1485 (24\%) deaths over a maximum follow-up of 6 years. The death rate was higher in HF versus no HF (11.49 vs 7.29 per 100 patient-years follow-up, $p<0.0001)$. Patients with $\mathrm{HF}$ and an NT-proBNP $>2000 \mathrm{pg} / \mathrm{mL}$ had lower survival than those with NT-proBNP $400-2000 \mathrm{pg} / \mathrm{mL}$ (3.8 years vs 5 years, $p<0.0001)$. Propensity matched survival curves were comparable between HFpEF and $\operatorname{HFrEF}(p=0.88)$. Conclusion Our findings support the use by NICE's HF diagnostic algorithm of tiered triage of patients with suspected HF based on their NT-proBNP levels. The two pathways yielded distinctive groups of patients with varied diagnoses and prognosis. HFpEF is the most frequent diagnosis, with its challenges of poor prognosis and paucity of therapeutic options.

\section{INTRODUCTION}

Heart failure (HF) is common (26 million patients worldwide) and is one of the leading causes of morbidity and mortality. ${ }^{1}$ HF predominantly affects people aged 50 years and beyond, ${ }^{2}$ and adversely affects patients' quality of life and survival. It poses an economic burden on the National Health Service of the UK. ${ }^{3}$ Early and accurate diagnosis and management of $\mathrm{HF}$ are paramount

\section{Key questions}

What is already known about this subject?

- Since the publication of the Chronic Heart Failure National Institute for health and Care Excellence (NICE) guidelines of 2010, which was updated in 2018, there was no published demonstration of its application in the real world until this year 2020. Recently, colleagues from Portsmouth and Southampton published their experience with the application of the diagnostic algorithm of these guidelines in a smaller cohort than ours. Their experience suggested that $53 \%$ of the patients presented with $\mathrm{N}$-terminal pro-brain-type natriuretic peptide (NT-proBNP) $>2000 \mathrm{pg} / \mathrm{mL}$. The diagnostic yield of NICE's NT-proBNP threshold in their cohort was $55 \%$, much lower than expected from the research literature. They found no difference in the survival between those with and without heart failure.

What does this study add?

- Our study reports on an almost 8-year experience with a large consecutive cohort of patients reflecting the incidence of heart failure in the community in Sheffield, an English city with 551800 inhabitants. We believe that our findings provide an insight into the real-world incidence of heart failure in the community, and demonstrate the value of the diagnostic algorithm suggested by NICE guidelines.

- Our study showed the diagnostic yield of NICE's NT-proBNP threshold $(400 \mathrm{pg} / \mathrm{mL})$ is $71 \%$, with the majority of the patients presenting with NT-pro BNP of $400-2000 \mathrm{pg} / \mathrm{mL}$. The study provides an insight into the survival rate of the different heart failure phenotypes and looks into the impact of the height of NT-proBNP, the symptom burden stipulated by the New York Heart Association functional class and the stages of chronic kidney disease on the prognosis. The study showed that there is no difference in the survival rate between those with heart failure and reduced or preserved ejection fraction, similar to the experience shown in acute heart failure setting among hospitalised patients in the UK National Heart Failure audit.

to alleviate symptoms, improve prognosis and lead to more cost-effective healthcare delivery. ${ }^{4}$ 


\section{Key questions}

How might this impact on clinical practice?

- The data we provide demonstrate the prominence of heart failure with preserved left ventricular ejection fraction as the most common diagnosis encountered in the community. This particular syndrome remains deprived of any evidence-based effective therapy. This calls on the medical and scientific community to concentrate the efforts into further research into the patho-physiology, prevention and treatment of the patients with this heart failure phenotype.

The National Institute for health and Care Excellence (NICE) produced a diagnostic algorithm for the patients suspected of HF. ${ }^{5}$ Thus, patients are referred for transthoracic echocardiography (TTE) and specialist assessment based on clinical presentation and measurement of N-terminal pro-brain-type natriuretic peptide (NT-proBNP). The diagnostic algorithm recommends patients are seen and assessed within 2 weeks if NT-proBNP $>2000 \mathrm{pg} / \mathrm{mL}$ and within 6 weeks if NT-proBNP is $400-2000 \mathrm{pg} / \mathrm{mL}^{5}$

Although the guidelines were published 10 years ago, limited data are available on the performance of the diagnostic algorithm. ${ }^{6}$ Reliable and contemporary description and characterisation of the population with HF is important to develop insight into HF trends, recognise clinical outcomes, and to improve and shape both current services and future clinical HF pathways.

The main objectives of this study are: first, to characterise real-world patients presenting to a HF clinic serving the city of Sheffield in the UK; second, to stratify patients whose diagnosis followed the established NICE HF diagnostic algorithm; and finally, to explore and investigate predictors of all-cause mortality in subphenotypes of HF defined in line with NICE guidelines 2010 (CG108) and its update in 2018 $(\mathrm{NG106)})^{57}$

\section{METHODS}

\section{Data and patients}

Patients suspected of HF from a population of 551800 are screened by their general practitioners (GPs) using a single measurement of NT-proBNP. In accordance with NICE guidelines, those with symptoms and/ or signs suggestive of $\mathrm{HF}$ and a raised NT-proBNP (threshold $400 \mathrm{pg} / \mathrm{mL}$ ) are referred to the HF clinic in Sheffield. ${ }^{57}$ Data were collected prospectively and electronically encrypted with an annual data validation check. The analytical cohort was derived from all patients presenting to this HF clinic between 13 April 2012 and 31 January 2020. The inclusion criteria were: age 18 years or over, raised NT-proBNP $(\geq 400 \mathrm{pg} / \mathrm{mL})$ and symptoms and/or signs suggestive of HF. Exclusion criteria were: incomplete TTE study, or absence of NT-proBNP measurement.

\section{HF assessment}

All patients referred to the HF clinic by their GPs with NT-proBNP $\geq 400 \mathrm{pg} / \mathrm{mL}$ underwent a resting 12-lead ECG and TTE. Each patient was clinically assessed by a specialist. The final diagnosis was determined by the HF specialist integrating the presenting history, clinical examination and the results of investigations in keeping with NICE guidelines. ${ }^{58}$

The patients with HF were subdivided into five phenotypes: HF with preserved ejection fraction (HFpEF), $\mathrm{HF}$ with reduced ejection fraction (HFrEF), HF due to pulmonary hypertension (HF-PH) where the only cardiac abnormality is pulmonary artery pressure of $\geq 40 \mathrm{~mm} \mathrm{Hg}$, HF due to right ventricular systolic dysfunction (HFRVSD) where the only cardiac abnormality is the systolic impairment of the right ventricle, and HF due to valvular heart disease (HF-VHD) where severe valvular disease is the only abnormality responsible for the syndrome of HF. It is recognised that pulmonary hypertension, RVSD and severe valvular disease could coexist with either HFrEF or HFpEF. In those circumstances, the patients were assigned as having HFrEF or HFpEF, respectively.

\section{Study variables}

Clinical and TTE variables are detailed in online supplemental file. The 12-lead ECG documented: rhythm (sinus, atrial fibrillation, atrial flutter or paced), QRS duration, left axis deviation, ST/T-wave changes, presence of ectopic beats, any conduction delays or atrioventricular blocks and presence of leftventricle (LV) hypertrophy using the standard voltage criteria. ${ }^{9}$ Similarly, details of the TTE findings were recorded in the database.

\section{Statistical analysis}

Statistical analyses were performed in MedCalc V.19.0.5. Categorical baseline characteristics are described with numbers and percentages. Continuous variables are described using means and SD. Comparison of continuous variables was done using an independent t-test, while the comparison of categorical variables was done using $\mathrm{X}^{2} \mathrm{t}$-test. Mortality rates are presented as per 100 patient-years (100PY) of follow-up (FU). Kaplan-Meier (KM) curves were used to visualise and interpret the data of variables associated with mortality. KM curves used the log-rank test to investigate the differences in curves between alive and dead patients at FU. Further survival analysis was done using propensity score matching for all clinical demographics which demonstrated association to all-cause mortality. In addition to propensity matched score method, we also tested the difference of survival curves between HFpEF and HFrEF using Cox's proportional hazard model. ${ }^{10}$ The Kruskal-Wallis one-way analysis of variance test was used to investigate the overall differences in clinical characteristics, the New York Heart Association (NYHA) symptom burden and chronic kidney disease (CKD) distribution of different subphenotypes of HF. All tests were two-sided, and statistical significance was considered if $\mathrm{p}$ value was $<0.05$. 
Table 1 Study demographics

\begin{tabular}{|c|c|c|c|}
\hline & \multirow{2}{*}{$\begin{array}{l}\text { No HF } \\
\mathrm{n}=1776\end{array}$} & \multirow{2}{*}{$\begin{array}{l}\text { HF } \\
n=4368\end{array}$} & \multirow[b]{2}{*}{$P$ value } \\
\hline & & & \\
\hline Age (years) & $78 \pm 9$ & $80 \pm 9$ & $<0.01$ \\
\hline NT-proBNP & $946 \pm 810$ & $2593 \pm 8918$ & $<0.01$ \\
\hline Gender (male) & $764(43 \%)$ & $1999(46 \%)$ & 0.05 \\
\hline Ischaemic heart disease & $406(23 \%)$ & $1565(36 \%)$ & $<0.01$ \\
\hline Valvular heart disease & $127(7 \%)$ & $1042(24 \%)$ & $<0.01$ \\
\hline Previous myocardial infarction & $125(7 \%)$ & $701(16 \%)$ & $<0.01$ \\
\hline Obesity & $57(3 \%)$ & $189(4 \%)$ & 0.043 \\
\hline Hypertension & $1110(63 \%)$ & $2856(65 \%)$ & 0.032 \\
\hline Diabetes mellitus & $307(17 \%)$ & $982(22 \%)$ & $<0.01$ \\
\hline Hypercholesterolaemia & $300(17 \%)$ & $906(21 \%)$ & $<0.01$ \\
\hline $\begin{array}{l}\text { Chronic obstructive pulmonary } \\
\text { disease }\end{array}$ & $328(18 \%)$ & $806(18 \%)$ & 0.988 \\
\hline Dementia & $18(1 \%)$ & $79(2 \%)$ & 0.023 \\
\hline Smoker & $507(29 \%)$ & $1358(31 \%)$ & 0.049 \\
\hline Cerebrovascular accident & $189(11 \%)$ & $598(14 \%)$ & $<0.01$ \\
\hline Chronic kidney disease (II-V) & $886(50 \%)$ & $2621(60 \%)$ & $<0.01$ \\
\hline $\begin{array}{l}\text { New York Heart Association } \\
\text { functional class }\end{array}$ & $1.8 \pm 0.7$ & $2.3 \pm 0.7$ & $<0.01$ \\
\hline
\end{tabular}

HF, heart failure; NT-proBNP, N-terminal pro-brain-type natriuretic peptide.

\section{RESULTS}

\section{Patient characteristics}

The demographic data and patient characteristics are summarised in tables 1 and 2. In almost 8 years' duration, 6272 patients were assessed in our HF clinic. From the total cohort, 128 (2\%) patients had other disorders (non-cardiac such as pulmonary fibrosis and cancer, and cardiovascular such as atrial arrhythmias, and pure valvular disease such as aortic stenosis without HF) and were excluded from further analysis. The study cohort was thus 6144 patients. Of this referred cohort of patients, 4368 patients $(71 \%)$ were diagnosed with HF and 1776 patients (29\%) did not have HF. Patients with HF were older $(80 \pm 9$ years vs $78 \pm 9$ years, $\mathrm{p}<0.01)$, had higher mean NT-proBNP levels $(2593 \pm 8918 \mathrm{pg} / \mathrm{mL}$ vs $946 \pm 810 \mathrm{pg} / \mathrm{mL}, \mathrm{p}<0.01)$ and had higher NYHA functional class $(2.3 \pm 0.7$ vs $1.8 \pm 0.7, \mathrm{p}<0.01)$ than the patients with no HF. Women comprised $54 \%$ of those with HF. For the total population, the mean NT-proBNP was $2114.4 \pm 7501 \mathrm{pg} / \mathrm{mL}$.

Compared with the patients with no HF, those with HF had higher prevalence of comorbidities such as systemic hypertension (65\% p=0.032), CKD $(60 \%$ $\mathrm{p}<0.01)$ and ischaemic heart disease $(36 \% \mathrm{p}<0.01)$. Similarly, more patients with HF had VHD, previous myocardial infarction (MI), diabetes mellitus (DM) and hypercholesterolaemia $(\mathrm{p}<0.01)$. Nearly one-third of the referred patients were smokers $(28 \%$ of those with no HF vs $38 \%$ of those with HF). On the other hand, there was no statistically significant difference in the incidence of chronic obstructive pulmonary disease (COPD) between patients with HF and without HF. Surprisingly, obesity was not a statistically significant comorbidity in any of the phenotypes of patients with HF.

\section{NT-proBNP >2000pg/mL vs NT-proBNP 400-2000pg/mL}

Patients with NT-proBNP $>2000 \mathrm{pg} / \mathrm{mL}$, using the 2-week pathway of the diagnostic algorithm, were 1624 patients

Table 2 Patient characteristics in various heart failure subcategorisations

\begin{tabular}{lccccccc}
\hline & HFpEF & HFrEF & HF-VHD & HF-PH & HF-RVSD & $\chi^{2}$ & P value \\
\hline $\mathrm{n}$ & 2022 & 1759 & 240 & 289 & 58 & & \\
Age (years) & $81 \pm 8$ & $78 \pm 10$ & $81 \pm 9$ & $78 \pm 10$ & $79 \pm 9$ & & $<0.01$ \\
NT-proBNP & $1780 \pm 3111$ & $3603 \pm 13450$ & $2698 \pm 3628$ & $2104 \pm 2588$ & $2309 \pm 5051$ & & $<0.01$ \\
\hline Sex (male) & $684(34 \%)$ & $1057(60 \%)$ & $93(39 \%)$ & $128(44 \%)$ & $37(64 \%)$ & 274 & $<0.01$ \\
IHD & $552(27 \%)$ & $881(50 \%)$ & $54(23 \%)$ & $66(23 \%)$ & $12(21 \%)$ & 265 & $<0.01$ \\
VHD & $354(18 \%)$ & $398(23 \%)$ & $220(92 \%)$ & $53(18 \%)$ & $17(29 \%)$ & 660 & $<0.01$ \\
MI & $179(9 \%)$ & $473(27 \%)$ & $21(9 \%)$ & $22(8 \%)$ & $6(10 \%)$ & 257 & $<0.01$ \\
Obesity & $100(5 \%)$ & $64(4 \%)$ & $11(5 \%)$ & $12(4 \%)$ & $2(3 \%)$ & 4 & 0.39 \\
Hypertension & $1469(73 \%)$ & $1022(58 \%)$ & $141(59 \%)$ & $192(66 \%)$ & $32(55 \%)$ & 96 & $<0.01$ \\
Diabetes mellitus & $493(24 \%)$ & $381(22 \%)$ & $43(18 \%)$ & $54(19 \%)$ & $11(19 \%)$ & 11 & 0.03 \\
Hypercholesterolaemia & $473(23 \%)$ & $357(20 \%)$ & $28(12 \%)$ & $41(14 \%)$ & $7(12 \%)$ & 31 & $<0.01$ \\
COPD & $347(17 \%)$ & $309(18 \%)$ & $37(15 \%)$ & $98(34 \%)$ & $15(26 \%)$ & 53 & $<0.01$ \\
Dementia & $35(2 \%)$ & $33(2 \%)$ & $3(1 \%)$ & $8(3 \%)$ & $0(0 \%)$ & 3 & 0.54 \\
Smoker & $654(32 \%)$ & $519(30 \%)$ & $63(26 \%)$ & $108(37 \%)$ & $14(24 \%)$ & 13 & 0.01 \\
CVA & $277(14 \%)$ & $255(14 \%)$ & $29(12 \%)$ & $30(10 \%)$ & $7(12 \%)$ & 4 & 0.36 \\
\hline
\end{tabular}

COPD, chronic obstructive pulmonary disease; CVA, cerebrovascular accident; HF, heart failure; HFpEF, heart failure with preserved ejection fraction; HFrEF, heart failure with reduced ejection fraction; IHD, ischaemic heart disease; MI, myocardial infarction; NT-proBNP, N-terminal pro-brain-type natriuretic peptide; $\mathrm{PH}$, pulmonary hypertension; RVSD, right ventricular systolic dysfunction; VHD, valvular heart disease. 


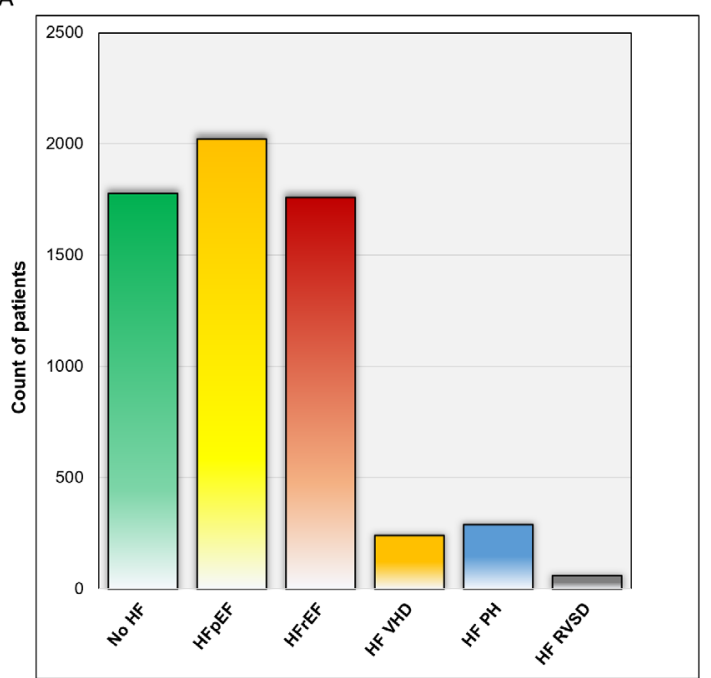



Figure 1 Final diagnosis in heart failure clinics for the whole population. (A) Histogram of the number of patients in each category of diagnosis. From a total of 6144 patients seen in the HF clinic, $29 \%$ of patients did not have HF. (B) In patients with NT-proBNP > 2000pg/ml, HFrEF was the main presenting diagnosis (50\%) which was followed by HFpEF (31\%).HF, heart failure; HFpEF, HF with preserved ejection fraction; HF-PH, HF with pulmonary hypertension; HFrEF, HF with reduced ejection fraction; HF-RVSD, HF with right ventricular systolic dysfunction; HF-VHD, HF with valvular heart disease; NT-proBNP, Nterminal pro-brain-type natriuretic peptide.

(26.4\% of the referred cohort). The remaining 4520 patients (73.6\%), who had NT-proBNP 400-2000 pg/mL, used the 6-week pathway.

Patients with NT-proBNP $>2000 \mathrm{pg} / \mathrm{mL}$ had a higher chance of having HF compared with those with NT-proBNP of $400-2000 \mathrm{pg} / \mathrm{mL}$ (92\% vs $64 \%$, respectively) (figure 1). Patients with HFpEF were similarly distributed between the 2-week and 6-week pathways ( $31 \%$ and $34 \%$, respectively). The similarity in distribution between those with NT-proBNP of 400-2000 pg/mL and those with NT-proBNP $>2000 \mathrm{pg} / \mathrm{mL}$ was also noted in those with HF-PH, HF-VHD and those with HF-RVSD. On the other hand, HFrEF was the single most common HF diagnosis $(50 \%)$ among the patients with NT-proBNP of $>2000 \mathrm{pg} / \mathrm{mL}$.

\section{Phenotypes of HF}

Table 2 shows the characteristics of the five phenotypes of the population with HF. Patients with HFrEF are significantly younger than patients with $\mathrm{HFpEF}$ and patients with HF-VHD. Of the 6144 referred cohort, HFpEF was the most common phenotype seen in 2022 patients (33\%), whereas HFrEF affected 1759 patients (29\%). Patients with HFpEF had a lower mean NT-proBNP levels $(1780 \pm 311 \mathrm{pg} / \mathrm{mL})$ than those with $\mathrm{HFrEF}$ $(3603 \pm 1345 \mathrm{pg} / \mathrm{mL})$ or HF-VHD $(2698 \pm 362 \mathrm{pg} / \mathrm{mL})$. Male patients predominated among those with $\mathrm{HFrEF}$ $(60 \%)$ and with HF-RVSD (64\%). There was female predominance in those with HFpEF (66\%), HF-VHD $(61 \%)$ or HF-PH $(56 \%)$.

\section{Symptom burden in HF phenotypes}

With an increase in HF symptom burden, HFrEF, HF-PH and HF-RVSD become more prevalent $(\mathrm{p}<0.0001)$ (figure 2A). HF-RVSD and HF-PH are more common in the patients presenting in NYHA functional class IV than those presenting in NYHA functional classes I-III. HFpEF is the diagnosis of the largest group of patients with NYHA classes I and II (48\% and 49\%, respectively), while representing only 23\% of those presenting with NYHA functional class IV. This suggests that the majority of the patients with HFpEF have milder symptoms. In contrast, HFrEF is the diagnosis in $50 \%$ of the patient cohort in NYHA functional class IV.

\section{The HF phenotypes in different stages of renal dysfunction (CKD)}

HFpEF was the diagnosis of $74 \%$ of the patients with HF and CKD stage $\mathrm{V}$ (figure 2B). In contrast, patients with HFrEF constituted $34 \%-43 \%$ of those with the other CKD stages. However, it is important to note that of the complete cohort, CKD stage $\mathrm{V}$ only represented $0.6 \%$ of the population (online supplemental figure 1). Thus, the observed increased incidence of HFpEF in CKD V is unlikely to have an impact on the outcomes of the cohort.

\section{Survival analysis}

Of the referred cohort, there were 1485 (24\%) deaths over a maximum FU period of 6 years (mean FU 2.3 \pm 2 years). The death rate was significantly higher in patients with HF (11.5 (95\% CI 11 to 12.2 ) vs 7.3 (95\% CI 6.5 to 8.2) per 100PY, $\mathrm{p}<0.0001)$.

The all-cause mortality as measured per $100 \mathrm{PY}$ of the patients with HF demonstrated an ascending order of the HF phenotypes: HFpEF (10.3 (95\% CI 9.4 to 11.3) per 100PY), HFrEF (11.8 (95\% CI 10.8 to 12.8 ) per 100PY), HF-RVSD (13.3 (95\% CI 7.5 to 22) per 100PY), HF-VHD (15 (95\% CI 11.8 to 18.7$)$ per 100PY) and the highest was 


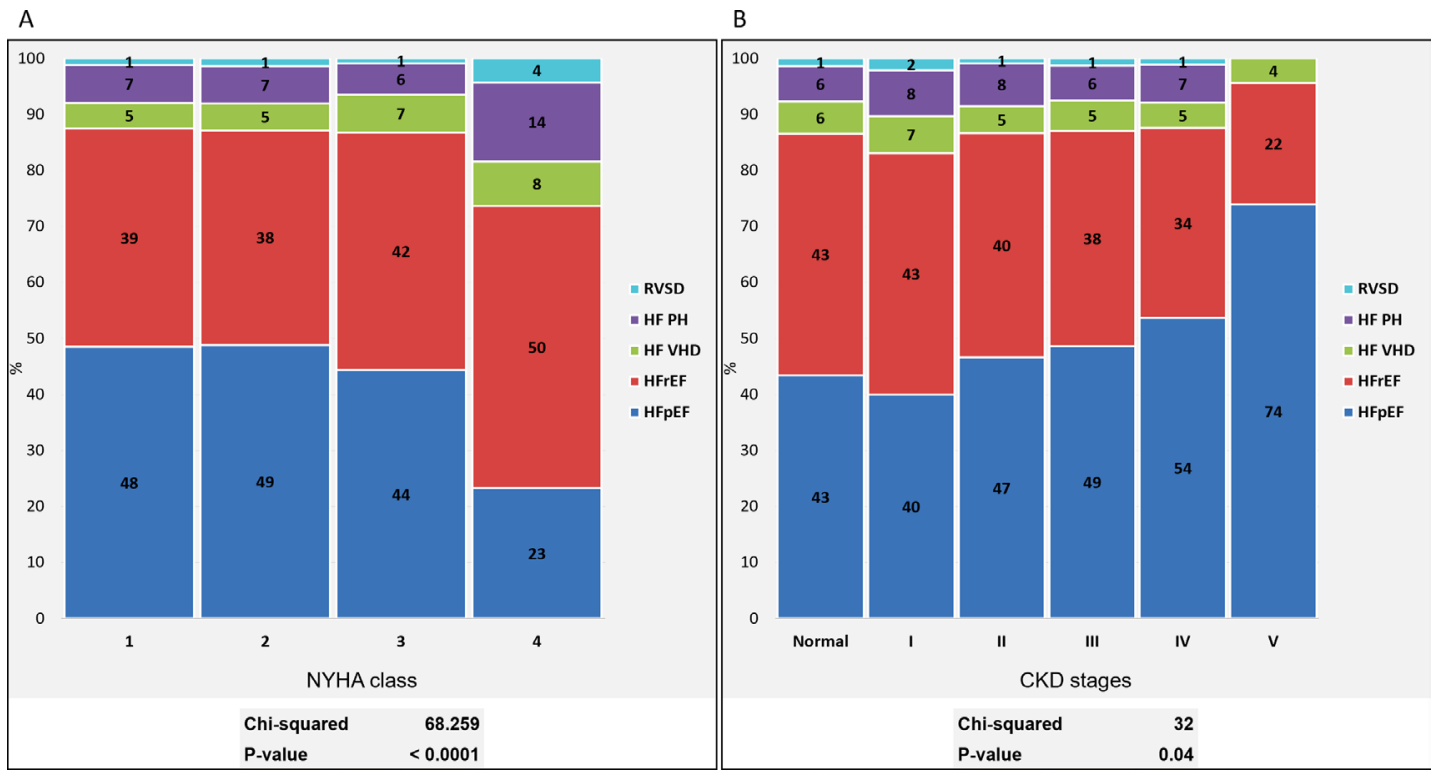

Figure 2 Stacked histogram demonstrating the percentage of patients with a specific HF diagnosis in each category of NYHA and CKD stage. (A) Patients with HFpEF were predominantly in NYHA functional class I/II, versus patients with HFrEF, who were predominantly in NYHA functional class IV. (B) HF patients with a diagnosis of HFpEF were more likely to have worse CKD stage than any other type of HF diagnosis. CKD, chronic kidney disease; HFpEF, heart failure with preserved ejection fraction; $\mathrm{HF}-\mathrm{PH}$, heart failure with pulmonary hypertension; HFrEF, heart failure with reduced ejection fraction; NYHA, New York Heart Association; RVSD, right ventricular systolic dysfunction; VHD, valvular heart disease

HF-PH (15.7 (95\% CI 12.9 to 19) per 100PY). There was no statistically significant difference between the mortality of those with HF-PH, HF-VHD or HF-RVSD. However, the differences were significant between those with HFpEF and HFrEF; and between each of those and the categories with

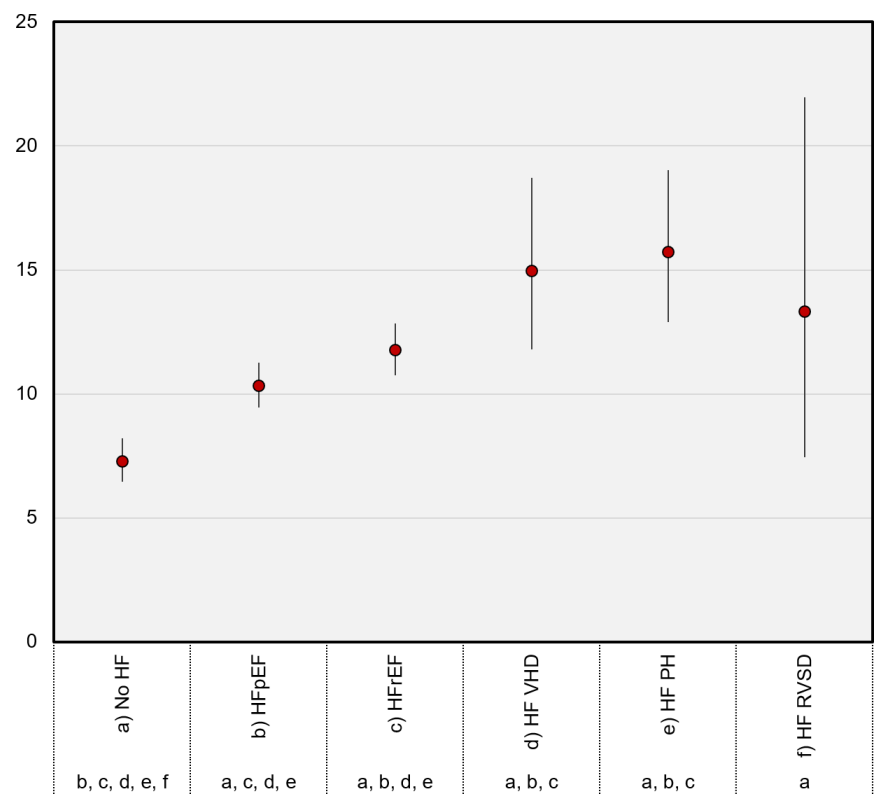

Figure 3 Incidence of all-cause mortality in each subphenotype of heart failure per 100 patient-years. Statistical significant changes to other groups are highlighted with letters representing the specific group. The lines represent the $95 \%$ confidence interval. HF, heart failure; HFpEF, HF with preserved ejection fraction; HF-PH, HF with pulmonary hypertension; HFrEF, HF with reduced ejection fraction; HF-RVSD, HF with right ventricular systolic dysfunction; HFVHD, HF with valvular heart disease. the highest mortality (HF-PH and HF-VHD) (figure 3). The median age for those with $\mathrm{HFpEF}$ was $81 \pm 8$ years of age and the median age of those with HFrEF being $78 \pm 10$ years of age $(p<0.01)$. However, since the patients with HFrEF are not particularly young, this difference may not have a significant biological difference.

On KM analysis, the survival curves were wide apart between those with no HF versus the patients with $\mathrm{HF}$ over the long- term FU period $\left(\chi^{2}=47.14, p<0.0001\right)$ (figure $4 \mathrm{~A}$ ). Whereas, the survival curves were not significantly different between HFpEF and HFrEF $\left(\chi^{2}=3.63, p=0.06\right)$.

Patients with HF and an NT-proBNP $\geq 2000 \mathrm{pg} / \mathrm{mL}$ had significantly worse survival rate than patients with lower NT-proBNP levels (3.8 years vs 5 years, $\chi^{2}=173.5$, $\mathrm{p}<0.0001$ ) (figure 5).

Worsening NYHA functional class was similarly associated with worsening mean survival (NYHA I: 4.8 years; NYHA II: 4.9 years; NYHA III: 4.2 years and NYHA IV: 2.47 years, $\chi^{2}=221.3, p<0.0001$ ) (figure $5 \mathrm{~B}$ ). On KM analysis, the obvious deterioration in survival rate started with NYHA functional class III and became marked with NYHA functional class IV.

For comparing patients with HF versus those with no $\mathrm{HF}$, and for comparing those with HFpEF versus those with HFrEF, the following covariates demonstrated association to outcome and were used in propensity matching: age, NT-proBNP level, male gender, obesity, hypertension, DM, hypercholesterolaemia, COPD, dementia, VHD, MI, stroke and renal impairment.

When comparing the survival of patients in different NYHA functional classes, all of the above variables were included for propensity matching, except NT-proBNP level that was replaced by phenotype diagnosis. 


\section{A}

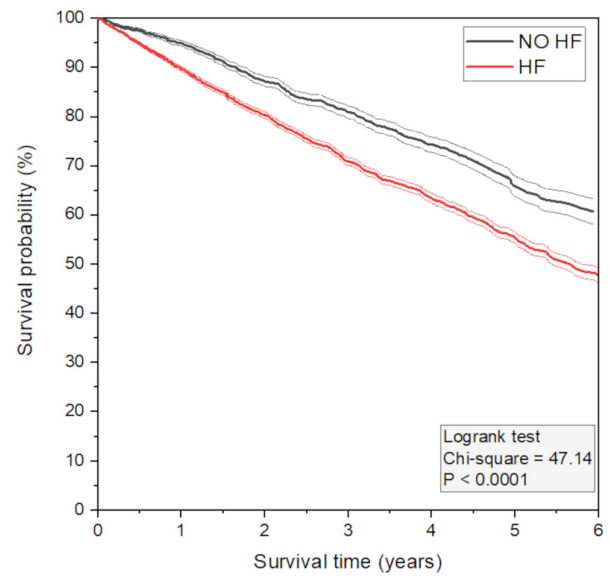

Number at risk

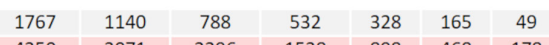

B

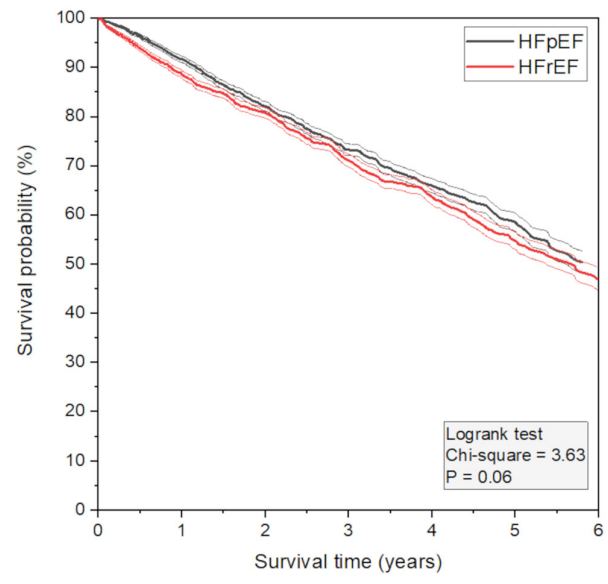

Number at risk

\begin{tabular}{|l|l|l|l|l|l|l|}
2016 & 1484 & 1088 & 687 & 352 & 190 & 88 \\
\hline 1752 & 1205 & 933 & 664 & 425 & 220 & 72 \\
\hline
\end{tabular}

C

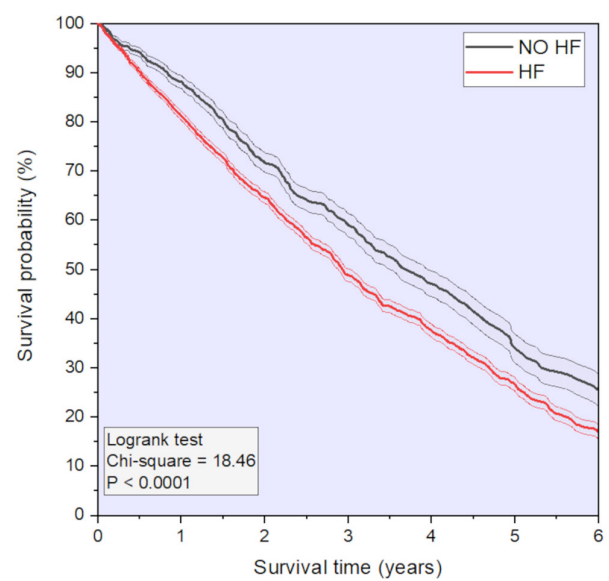

Number at risk

\begin{tabular}{|c|c|c|c|c|c|c|}
\hline 694 & 474 & 306 & 194 & 118 & 56 & 19 \\
\hline 2123 & 1482 & 1025 & 628 & 361 & 171 & 52 \\
\hline
\end{tabular}

D

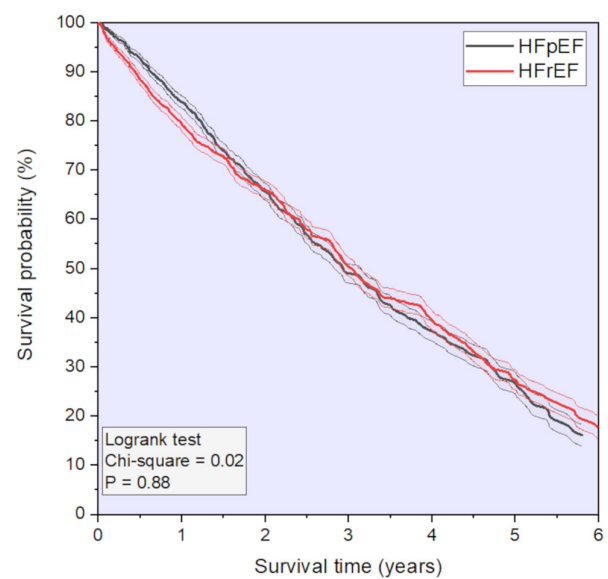

Number at risk

\begin{tabular}{l|l|l|l|l|l|l|}
936 & 676 & 441 & 253 & 131 & 63 & 23 \\
\hline 858 & 590 & 444 & 287 & 180 & 86 & 22 \\
\hline
\end{tabular}

Figure 4 Kaplan-Meier survival curves. (A and B) Survival comparison of patients with versus without HF and with HFpEF versus HFrEF, over a follow-up period of up to 6 years. (C and D) Propensity matched survival comparison of patients with versus without $\mathrm{HF}$ and with $\mathrm{HFpEF}$ versus $\mathrm{HFrEF}$, over a follow-up period of up to 6 years. HF, heart failure; HFpEF, HF with preserved ejection fraction; HFrEF, HF with reduced ejection fraction.

Post-propensity matching, the differences between the survival curves were less for the comparison of patients with no HF versus patients with HF than those seen on $\mathrm{KM}$ analysis before propensity matching, but remained statistically significantly different $\left(\chi^{2}=18.46, \mathrm{p}<0.0001\right)$ (figure 4C). The survival curves of HFpEF and HFrEF almost overlap demonstrating no actual difference in survival over time $\left(\chi^{2}=0.02, p=0.88\right)$ (figure $4 D$ ). By adjusting for covariates using the Cox's proportional hazard model, the survival trend was worse for HFrEF, but this again remained statistically not significant (figure 6). The differences in survival curves remained statistically significantly different for both NT-proBNP and NYHA functional class after propensity matching (figure 5).

\section{DISCUSSION}

Our study sought to characterise the patients referred from the community to the HF clinic using NICE diagnostic algorithm ${ }^{5}$ and to investigate their outcomes. Until this year 2020, these have remained largely untested.

We prospectively entered all patients referred to the HF clinic into the SHEAF registry. ${ }^{11}$ We excluded the few patients before 2013 whose entry into the diagnostic algorithm was through a history of MI. We observed that 


\section{A}
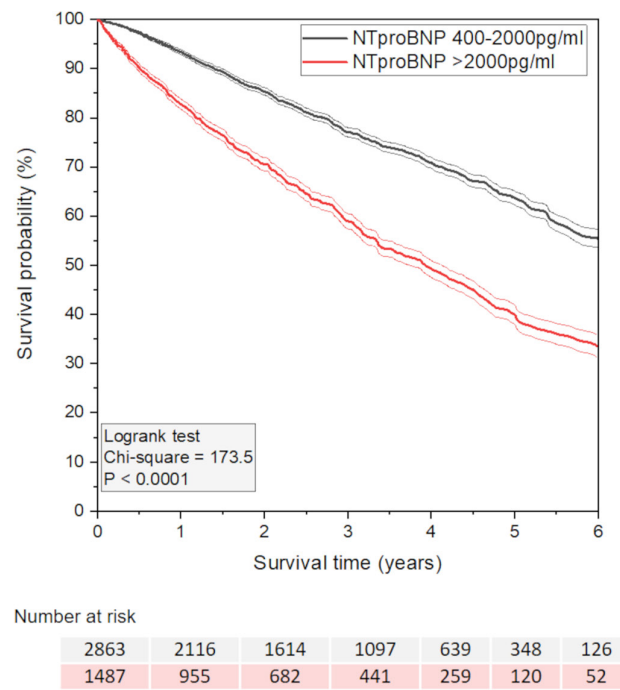

C

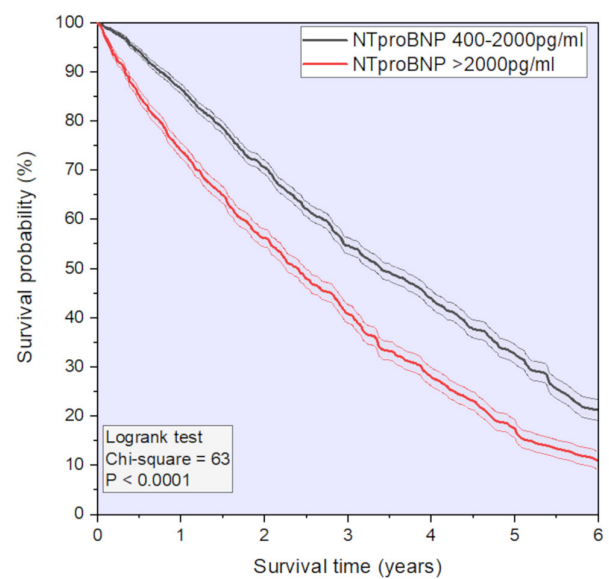

Number at risk

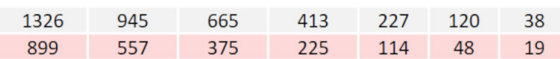

B
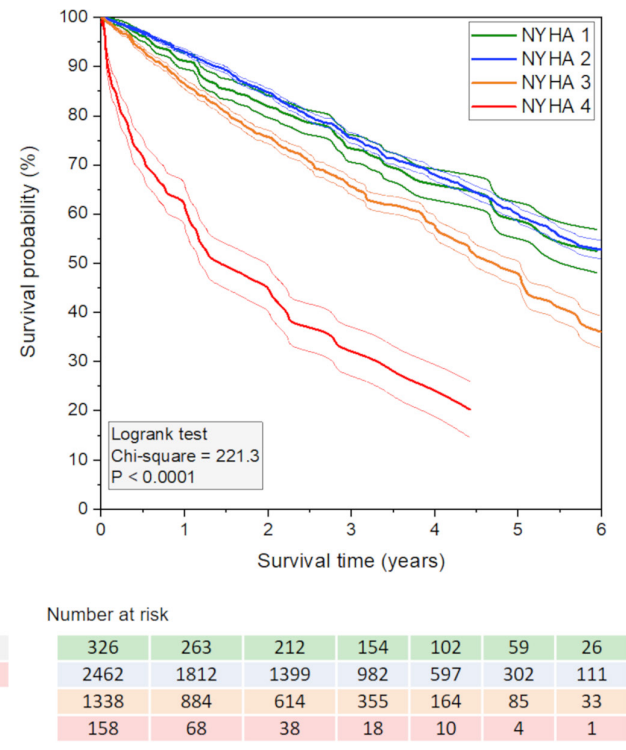

D

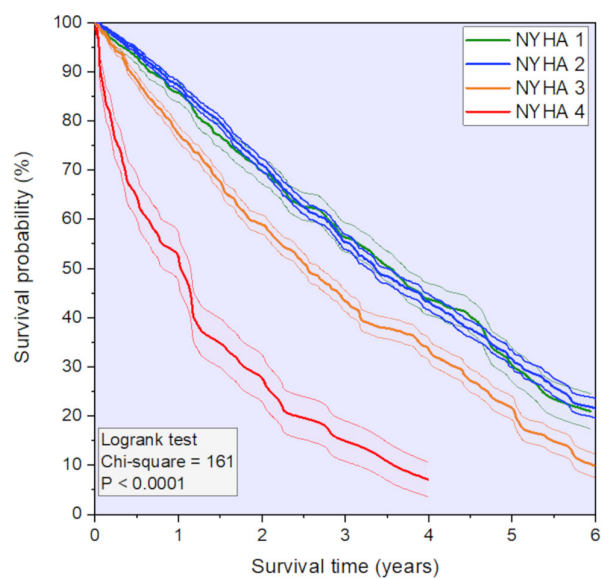

Number at risk

\begin{tabular}{|c|c|c|c|c|c|c|}
\hline 374 & 276 & 197 & 130 & 71 & 38 & 15 \\
\hline 1504 & 1063 & 759 & 494 & 296 & 133 & 38 \\
\hline 813 & 507 & 323 & 170 & 91 & 40 & 9 \\
\hline 116 & 45 & 22 & 8 & 3 & 2 & 1 \\
\hline
\end{tabular}

Figure 5 Kaplan-Meier survival curves. (A and B) Survival comparison of patients with NTproBNP 400-1999pg/ml versus $\geq$ NTproBNP 400-1999pg/ml, and in different NYHA functional status, over a follow-up period of up to 6 years. (C and D) Propensity matched survival comparison of patients with NTproBNP 400-1999pg/ml versus $\geq$ NTproBNP 400-1999pg/ml, and in different NYHA functional status, over a follow-up period of up to 6 years. NT-proBNP, N-terminal pro-brain-type natriuretic peptide; NYHA, New York Heart Association.

GPs tended to test the NT-proBNP even when there was a history of MI. Zheng $e t a t^{6}$ made a similar observation to ours. The majority of the patients with a history of MI referred to our HF clinic in that first year had NT-proBNP levels $<2000 \mathrm{pg} / \mathrm{mL}$. Thus, these patients were entered into the 6-week pathway. We have amended the protocol making raised NT-proBNP as the single port of entry into the diagnostic HF service. This modification was subsequently adopted by NICE in the chronic HF guidelines (NG106-2018). ${ }^{7}$

The most common phenotype of HF in the community patients in the SHEAF registry was HFpEF. Indeed, one of the step changes in the HF NICE guidelines (CG108) of 2010 was the introduction of the almost universal use of natriuretic peptides (NPs) by GPs as the main port of entry into the HF diagnostic algorithm. ${ }^{5}$ This key investigation to access HF diagnostic services in secondary care is based on its high specificity at the thresholds chosen. ${ }^{12} 13$ Prior to those guidelines, many cases of HFpEF were undiagnosed. Within the diagnostic algorithm, the referred patients are triaged into a 2-week and 6-week pathways based on the level of the NPs. This was due to the poor short-term and medium-term outcomes shown in patients with HF and very high NP levels. ${ }^{14}$ 


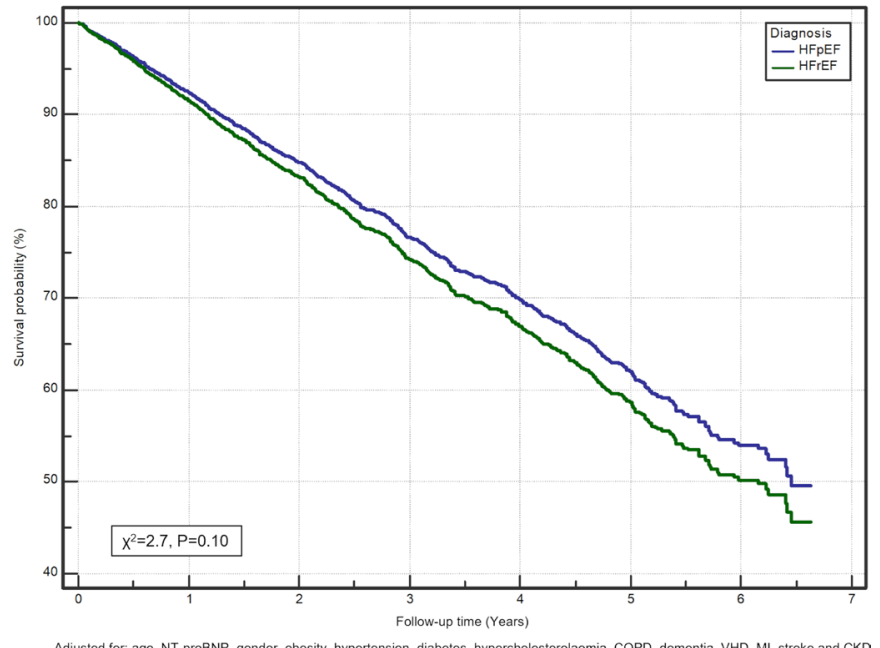

igure 6 Kaplan-Meier curves comparing HFpEF and HFrEF survival. These curves have been adjusted for covariates using the Cox's proportional-hazards regression. CKD, chronic kidney disease; COPD, chronic obstructive pulmonary disease; HFpEF, heart failure with preserved ejection fraction; HFrEF, heart failure with reduced ejection fraction; Ml; myocardial infarction; NT-proBNP, N-terminal pro-brain-type natriuretic peptide; VHD, valvular heart disease.

Thus, patients with NT-proBNP $>2000 \mathrm{pg} / \mathrm{mL}$ have their TTE and see the specialist within 2 weeks, while those with NT-proBNP $400-2000 \mathrm{pg} / \mathrm{mL}$ have the TTE and the specialist's review within 6 weeks. Providing patients with early specialist review to ensure the correct diagnosis is made and the appropriate management plan is implemented are known to improve outcomes. ${ }^{9}$

The HF NICE guidelines of 2010 (CG108) and of 2018 (NG106) chose the diagnostic threshold for NT-proBNP at $400 \mathrm{pg} / \mathrm{mL}$. This is at variance with the European Society of Cardiology (ESC) threshold of $125 \mathrm{pg} /$ $\mathrm{mL} .{ }^{15} 16$ The ESC approach is based on ruling out HF, at the expense of low specificity at that threshold. This approach can potentially overwhelm echocardiography services and specialist clinics. ${ }^{17}$ The cost-effectiveness of NICE approach of using NT-proBNP as a gatekeeper for the diagnosis of HF has been further confirmed by the work of Taylor et al..$^{717}$

We recognise that elevated NT-proBNP is an independent risk factor for cardiovascular events and mortality in the general population. ${ }^{18}$ Indeed, we demonstrated that those with no HF $(29 \%)$ suffered mortality rate at 7.3 (95\% CI 6.5 to 8.2 ) per 100PY. We have published the clinical predictors of mortality in this group of people. ${ }^{11}$ The patients diagnosed with HF had a significantly higher mortality rate of 11.5 (95\% CI 11 to 12.2 ) per $100 \mathrm{PY}$.

Biomarkers of myocardial fibrosis in patients with chronic HF (soluble ST2 receptor, galectin-3 and high sensitivity cardiac troponin) were proposed as additives to NP in aiding prognostication of patients with HF. ${ }^{19-21}$ Whether we adopt novel cardiac biomarkers as an adjunct to NT-proBNP in the triage or prognostication process remains to be seen.

Among our cohort of 6144 patients, 26.4\% entered the 2-week pathway (NT-proBNP $>2000 \mathrm{pg} / \mathrm{mL}$ ), while $73.6 \%$ entered the algorithm via the 6 -week pathway (NT-proBNP 400-2000 pg/mL). Those percentages follow a natural distribution pattern, but are at odds with those of Zheng et at as $53 \%$ of their patients used the 2-week pathway.

In our cohort, $71 \%$ of the patients with NT-proBNP $\geq 400 \mathrm{pg} / \mathrm{mL}$ had HF, which is close to the expected yield of diagnostic studies that formed the basis of NICE's choice of that threshold. ${ }^{513}$ Furthermore, there was a higher diagnostic yield $(92 \%)$ with the NT-proBNP $>2000 \mathrm{pg} / \mathrm{mL}$ versus a diagnostic yield of $64 \%$ when NT-proBNP is $400-2000 \mathrm{pg} / \mathrm{mL}$. It was interesting to note that in their recently published study, Zheng et at reported a low diagnostic yield of the same NT-proBNP threshold of $\geq 400 \mathrm{pg} / \mathrm{mL}$ at only $55 \%$.

While our study and that of Zheng et at have implemented the same diagnostic algorithm by NICE, ${ }^{5}$ our results appear to reflect potentially different incidence of HF in the community. Their two centres serve a population of 1175000 people. Portsmouth and Southampton contributed to their study for 35 months and 24 months, respectively. Their 1271 referrals suggested that Southampton received 2 referrals/100 000/month, and Portsmouth received 4 referrals/100 000/month. In contrast, in Sheffield we serve a population of 551800 , and received 6144 referrals in 93 months, at a mean rate of 12 referrals/100 000/month.

Notwithstanding the differences between our findings and those by Zheng et al, it is important to recognise that observational studies like ours and theirs are significantly affected by the epidemiological differences related to the different characteristics of the populations each of our groups serves, and the impact of referral patterns by primary care.

Unsurprisingly, the survival analysis of our larger registry showed the mortality rate is higher among our patients with $\mathrm{HF}$ versus those with no HF table 3(figure 4). While the two groups were similar in age and comorbidities, the main difference between them is the presence of any form of HF. Taylor $e t a t^{22}$ published a populationbased cohort study in the UK from primary care data of 55959 new diagnoses of HF over 17 years. The survival trends were significantly worse at 1 year, 5 years and 10 years in the HF versus no HF groups. These findings are consistent with what we observed in the SHEAF registry. In contrast, Zheng et at $t^{6}$ reported no difference in the survival between the HF versus no HF groups, which is surprising.

Other findings of interest are the impact on the prognosis of both the degree of rise in the NT-proBNP and the severity of symptoms at presentation as defined by the NYHA class. Those presenting with NT-proBNP $>2000 \mathrm{pg} / \mathrm{mL}$ survived on average 1.2 year less than those presenting with NT-proBNP of $400-2000 \mathrm{pg} / \mathrm{mL}$. 
Similarly, there was a 2.33 years difference in survival between those with NYHA functional class I at presentation and those presenting with NYHA functional class IV.

We have confirmed the findings of others ${ }^{23}$ as we demonstrated that those with HF-PH have an even higher mortality than those with HFpEF or HFrEF (figure 3).

Those who emphasise the impact of lower left ventricular ejection fraction (LVEF) on survival feel that patients with HFrEF should have higher mortality than those with HFpEF. Others may attach more emphasis on the impact of comorbidities such as advanced CKD or characteristics such as ageing. A third group may believe that the absence of effective therapy for patients with HFpEF should increase their mortality rate compared with the patients with HFrEF. In our study, the mortality at 100PY FU showed statistically significant difference between those with HFpEF and those with HFrEF. However, when we adjusted for covariates using the Cox's proportional hazard model (figure 6), the KM survival curves showed no statistically significant difference between the two groups. As our dataset is a large one, we have appropriately used propensity matched analysis representing real data instead of adjusting for the mean of covariates. The latter method produced KM survival curves of HFpEF and HFrEF that almost overlapped demonstrating no actual difference in survival over time (figure 4D). Thus, we confirmed there is no statistically significant difference between the survival of patients with these two phenotypes of HF over a maximum FU of 6 yearstable 3 .

Not unlike our findings, Ambrosy et $a t^{24}$ showed that while there is a trend toward lower in-hospital mortality among hospitalised patients with HF with HFpEF in the Get With The Guidelines-HF programme, FU data from the Organized Program to Initiate Lifesaving Treatment in Hospitalized Patients With HF registry suggest that post-discharge survival is poor irrespective of EF. In other words, when one extends the FU beyond the first 3 months, one would be surprised to find similar survival in both groups. Similarly, Varela-Roman et al demonstrated no effect of LVEF on the survival. ${ }^{25}$

These are similar to other studies including the results from the UK HF national audit. An older cohort studied between 1987 and 2001 showed small differences in the mortality of patients with HFpEF and patients with HFrEF, at 1 and 5 years $(29 \%$ vs $32 \%$, and $65 \%$ vs $68 \%$, respectively). ${ }^{26}$ The latter series predates the impact of major advances in therapeutics for HFrEF, with beta-blockers and mineralocorticoid antagonists. Thus, the improved survival of patients with HFrEF coupled with absence of disease-modifying therapy for $\mathrm{HFpEF}^{27}$ allowed the outcomes of these two phenotypes of HF to become similar.

We propose that a UK-wide diagnostic HF registry is established along the lines we established in the SHEAF registry in Sheffield. This will require several conditions to be met as described in a recent editorial in Heart. ${ }^{28}$

\section{Clinical perspective}

This is the largest published registry of an HF clinic in the UK and is the second description of the implementation of the diagnostic algorithm of NICE HF guidelines. ${ }^{57}$ The prospective entry of data by specialists in HF, and the fact the clinic serves the whole population of patients with suspected HF in one city improves our confidence that the characterisation of the patients enrolled is a true representation of such a population.

\section{Limitations}

There are four limitations: we are uncertain that all the patients reporting symptoms of HF were tested and referred, we do not know if there were patients suspected of HF with raised NT-proBNP who were not referred to the service, there were patients whose HF diagnosis was made when they were admitted acutely with HF bypassing our clinic, and finally we have not been able to provide detailed analysis of patients without complete TTE due to lack of data.

\section{CONCLUSION}

Our findings support the use by NICE's HF diagnostic algorithm of tiered triage of patients with suspected HF based on their NT-proBNP levels. The two pathways yielded distinctive groups of patients with varied diagnoses and prognoses. We recognise the eminence of $\mathrm{HFpEF}$ as the most frequent diagnosis, with its challenges of poor prognosis and paucity of therapeutic options. This demands of all of us to work harder to investigate the pathophysiology, course and potential management of HFpEF.

Twitter Hosamadin Assadi @HosamAssadi and Abdallah Al-Mohammad @ AAIMohammad87

Acknowledgements We thank the Sheffield Teaching Hospitals Heart Failure services in their pursuit to collect, record and manage the data used for this study.

Contributors PG and AA-M conceptualised and organised the study. GF, NL, DR, AC and $A A-M$ facilitated data collection. AJS and PG did all the statistical analyses. AJS facilitated the ethical approval. HA, UN and AD helped draft the initial manuscript. PG drafted and revised figures and tables. AA-M and PG provided critical input into the content and discussion regarding the findings of the study. All authors took part in critical review and drafting of the manuscript, and have read and approved the final manuscript.

Funding AJS is supported by Wellcome Trust (AS: 205188/Z/16/Z). PG is supported by the Academy of Sciences Starter Grant (PG: SGL018/1100).

Competing interests None declared.

Patient consent for publication Not required.

Ethics approval The Sheffield HEArt Failure registry (SHEAF registry) has been sanctioned by the local 3D-lab committee under the registration number 222349P4. This has the appropriate research ethics committee approval (17/ $\mathrm{YH} / 0142)$. This study complies with the Declaration of Helsinki.

Provenance and peer review Not commissioned; externally peer reviewed.

Data availability statement All data relevant to the study are included in the article or uploaded as online supplemental information.

Open access This is an open access article distributed in accordance with the Creative Commons Attribution Non Commercial (CC BY-NC 4.0) license, which permits others to distribute, remix, adapt, build upon this work non-commercially, and license their derivative works on different terms, provided the original work is 
properly cited, appropriate credit is given, any changes made indicated, and the use is non-commercial. See: http://creativecommons.org/licenses/by-nc/4.0/.

ORCID iDs

Pankaj Garg http://orcid.org/0000-0002-5483-169X

Hosamadin Assadi http://orcid.org/0000-0002-6143-8095

Abdallah Al-Mohammad http://orcid.org/0000-0001-6517-8692

\section{REFERENCES}

1 Crespo-Leiro MG, Anker SD, Maggioni AP, et al. European Society of Cardiology Heart Failure Long-Term Registry (ESC-HF-LT): 1-year follow-up outcomes and differences across regions. Eur J Heart Fail 2016;18:613-25.

2 Mosterd A, Hoes AW. Clinical epidemiology of heart failure. Heart 2007;93:1137-46.

3 von Lueder TG, Agewall S. The burden of heart failure in the general population: a clearer and more concerning picture. J Thorac Dis 2018;10:S1934-7.

4 Galinier M, Cariou E, Lairez O, et al. [Place of natriuretic peptides in the early diagnosis of heart failure in community medicine]. Presse Med 2018;47:804-10.

5 NICE. Chronic heart failure in adults: management; Guidance. Available: https://www.nice.org.uk/guidance/cg108

6 Zheng A, Cowan E, Mach L, et al. Characteristics and outcomes of patients with suspected heart failure referred in line with National Institute for health and care excellence guidance. Heart 2020;106:1579-85.

7 NICE. Chronic heart failure in adults: diagnosis and management. Available: https://www.nice.org.uk/guidance/ng106

8 Al-Mohammad A, Mant J, Laramee P, et al. Diagnosis and management of adults with chronic heart failure: summary of updated NICE guidance. BMJ 2010;341:c4130.

9 Casale PN, Devereux RB, Alonso DR, et al. Improved sex-specific criteria of left ventricular hypertrophy for clinical and computer interpretation of electrocardiograms: validation with autopsy findings. Circulation 1987;75:565-72.

10 Makuch RW. Adjusted survival curve estimation using covariates. $J$ Chronic Dis 1982;35:437-43.

11 Garg P, Wood S, Swift AJ, et al. Clinical predictors of all-cause mortality in patients presenting to specialist heart failure clinic with raised NT-proBNP and NO heart failure. ESC Heart Fail 2020;7:1791-800.

12 Roberts E, Ludman AJ, Dworzynski K, et al. The diagnostic accuracy of the natriuretic peptides in heart failure: systematic review and diagnostic meta-analysis in the acute care setting. BMJ 2015;350:h910.

13 Mant J, Doust J, Roalfe A, et al. Systematic review and individual patient data meta-analysis of diagnosis of heart failure, with modelling of implications of different diagnostic strategies in primary care. Health Technol Assess 2009;13:1-207.

14 Kubánek M, Goode KM, Lánská V, et al. The prognostic value of repeated measurement of $\mathrm{N}$-terminal pro-B-type natriuretic peptide in patients with chronic heart failure due to left ventricular systolic dysfunction. Eur J Heart Fail 2009;11:367-77.

15 McMurray JJV, Adamopoulos S, Anker SD, et al. ESC guidelines for the diagnosis and treatment of acute and chronic heart failure 2012: the task force for the diagnosis and treatment of acute and chronic heart failure 2012 of the European Society of cardiology. developed in collaboration with the heart failure association (HFA) of the ESC. Eur Heart J 2012;33:1787-847.

16 Ponikowski P, Voors AA, Anker SD, et al. 2016 ESC Guidelines for the diagnosis and treatment of acute and chronic heart failure: The Task Force for the diagnosis and treatment of acute and chronic heart failure of the European Society of Cardiology (ESC)Developed with the special contribution of the Heart Failure Association (HFA) of the ESC. Eur Heart J 2016;37:2129-200.

17 Taylor CJ, Roalfe AK, Iles R, et al. Primary care referral for echocardiogram (refer) in heart failure: a diagnostic accuracy study. Br J Gen Pract 2017;67:e94-102.

18 Mehta PA, Dubrey SW, Mclntyre HF, et al. Improving survival in the 6 months after diagnosis of heart failure in the past decade: population-based data from the UK. Heart 2009;95:1851-6.

19 Geng Z, Huang L, Song M, et al. N-terminal pro-brain natriuretic peptide and cardiovascular or all-cause mortality in the general population: a meta-analysis. Sci Rep 2017;7.

20 Piek A, Du W, de Boer RA, et al. Novel heart failure biomarkers: why do we fail to exploit their potential? Crit Rev Clin Lab Sci 2018;55:246-63.

21 Braunwald E. Biomarkers in heart failure. N Engl J Med 2008;358:2148-59.

22 Taylor CJ, Ordóñez-Mena JM, Roalfe AK, et al. Trends in survival after a diagnosis of heart failure in the United Kingdom 2000-2017: population based cohort study. BMJ 2019;13:I223.

23 Chatterjee NA, Lewis GD, Lewis Gregory D. What is the prognostic significance of pulmonary hypertension in heart failure? Circ Heart Fail 2011;4:541-5.

24 Ambrosy AP, Fonarow GC, Butler J, et al. The global health and economic burden of hospitalizations for heart failure: lessons learned from hospitalized heart failure registries. J Am Coll Cardiol 2014:63:1123-33.

25 Varela-Roman A, Grigorian L, Barge E. Heart failure in patients with preserved and deteriorated left ventricular ejection fraction. Heart 2005;91:489-94.

26 Owan TE, Hodge DO, Herges RM, et al. Trends in prevalence and outcome of heart failure with preserved ejection fraction. $N$ Engl Med 2006;355:251-9.

27 Ilieșiu AM, Hodorogea AS. Treatment of heart failure with preserved ejection fraction. Adv Exp Med Biol 2018;1067:67-87.

28 Al-Mohammad A. NICE heart failure guidelines: the diagnostic algorithm in practice, what is the next step? Heart 2020;106:1547-8. 